\title{
Identification of epigenetic signature associated with alpha thalassemia/mental retardation X-linked syndrome
}

\author{
Laila C. Schenkel ${ }^{1}$, Kristin D. Kernohan², Arran McBride², Ditta Reina ${ }^{8,9}$, Amanda Hodge $^{8,9}$, \\ Peter J. Ainsworth 1,3,4,5,6, David I. Rodenhiser ${ }^{4,5,6,7}$, Guillaume Pare ${ }^{8,9}$, Nathalie G. Bérubé ${ }^{4,5,6,7}$, Cindy Skinner ${ }^{10}$, \\ Kym M. Boycott ${ }^{2}$, Charles Schwartz ${ }^{10}$ and Bekim Sadikovic ${ }^{1,3,7^{*}}$
}

\begin{abstract}
Background: Alpha thalassemia/mental retardation X-linked syndrome (ATR-X) is caused by a mutation at the chromatin regulator gene ATRX. The mechanisms involved in the ATR-X pathology are not completely understood, but may involve epigenetic modifications. ATRX has been linked to the regulation of histone $\mathrm{H} 3$ and DNA methylation, while mutations in the ATRX gene may lead to the downstream epigenetic and transcriptional effects. Elucidating the underlying epigenetic mechanisms altered in ATR-X will provide a better understanding about the pathobiology of this disease, as well as provide novel diagnostic biomarkers.

Results: We performed genome-wide DNA methylation assessment of the peripheral blood samples from 18 patients with ATR-X and compared it to 210 controls. We demonstrated the evidence of a unique and highly specific DNA methylation "epi-signature" in the peripheral blood of ATRX patients, which was corroborated by targeted bisulfite sequencing experiments. Although genomically represented, differentially methylated regions showed evidence of preferential clustering in pericentromeric and telometric chromosomal regions, areas where ATRX has multiple functions related to maintenance of heterochromatin and genomic integrity.

Conclusion: Most significant methylation changes in the 14 genomic loci provide a unique epigenetic signature for this syndrome that may be used as a highly sensitive and specific diagnostic biomarker to support the diagnosis of ATR-X, particularly in patients with phenotypic complexity and in patients with ATRX gene sequence variants of unknown significance.
\end{abstract}

Keywords: ATRX, DNA methylation, Epi-signature, Intellectual disability, Biomarker

\section{Background}

An emerging development in the field of medical genetics has been the identification of Mendelian disorders involving genes encoding the writers, erasers, readers and remodelers of the epigenetic machinery [1]. Building on several decades of evidence regarding the functions of covalent DNA methylation $[2,3]$ and histone modifications [4] in regulating gene transcription, it is evident

\footnotetext{
*Correspondence: Bekim.Sadikovic@lhsc.on.ca

${ }^{3}$ Molecular Genetics Laboratory, Victoria Hospital, London Health

Sciences Center, 800 Commissioner's Road E, B10-104, London, ON N6A

5W9, Canada

Full list of author information is available at the end of the article
}

that mutations in the proteins responsible for creating, interpreting or removing the broad arrays of epigenetic marks can be linked to genetic conditions including cancer [5], imprinting disorders and/or single-gene disorders [6].

Along with these discoveries came the opportunity, not only for the elucidation of underlying molecular mechanisms altered in these disorders, but also for the identification of epi-signatures that can be diagnostically useful, specifically where patients express a subset of clinical manifestations associated with a phenotypic spectrum shared across more than one syndrome, making a specific clinical diagnosis difficult to reach. 
Among the rapidly expanding number of proteins responsible for chromatin maintenance and remodeling related to transcription is alpha thalassemia/mental retardation X-linked (ATRX; NG_008838.2). Mutations in the ATRX gene cause alpha thalassemia/mental retardation X-linked syndrome (ATR-X, OMIM 301040), a disorder characterized by moderate to severe intellectual disability, expressive language disorder, characteristic facial gestalt during infancy, often associated with hematological signs of alpha thalassemia [7].

The ATRX protein functions as an agent of ATPdependent chromatin remodeling and is a member of the SWI/SNF superfamily of proteins. The latter can have a wide variety of cellular functions, as described in detail in several recent reviews [8-10]. Briefly, ATRX protein is involved in cellular processes such as meiosis, mitosis, DNA repair and regulation of transcription through an effect on chromatin [11-15]. Disruption of these activities may contribute to developmental abnormalities associated with the ATR-X syndrome.

Within the ATRX protein, a histone-binding ATRXDNMT3-DNMT3L (ADD) domain can sense the methylation modifications of both H3K4 and H3K9 [16], essentially acting as an interpreter of these histone states. ATRX is also known to associate with the transcription cofactor DAXX. ATRX-DAXX complex is responsible for deposition of histone $\mathrm{H} 3.3$ at the telomeric and pericentromeric heterochromatic regions within chromosomes [17]. Loss of ATRX in ES cells leads to the loss of histone $\mathrm{H} 3.3$ at imprinting control regions and telomeric regions, along with the concurrent loss of H3K9me3 $[18,19]$. ATRX has also been linked to DNA methylation regulation, as mutations at the ATRX gene result in DNA methylation changes at subtelomeric and repetitive regions [20].

The role of ATRX as a regulator of heterochromatin dynamics raises the possibility that mutations in ATRX may lead to downstream transcriptional effects across the complex of genes or repetitive regions involved in the global context of the disorder, in addition to explaining phenotypical differences in these patients. For example, ATRX mutations affect the expression of $\alpha$-globin gene cluster, causing $\alpha$-thalassemia [21]. Mechanistically, $\alpha$-globin cluster, among other genes, has G-rich tandem repeats (TRs) sites, which have been reported to bind ATRX resulting in H3.3 deposition and gene expression regulation. In addition, it was suggested that the differences in size of these TRs among ATR-X patients contribute to the ranges in severity of the syndrome [22].

The orchestrated regulation of epigenetic mechanisms, including associations between ATRX and DNA methylation $[11,12]$, is essential for tissue homeostasis, cell identity and proper human development. Here, we describe the findings of a genome-wide DNA methylation array (GWMA) performed on peripheral blood samples from patients with ATR-X and show the genome-wide changes in DNA methylation that occur in patients with this epigenetic syndrome. We have identified a specific epi-signature of differentially hypo- and hypermethylated genes in patients clinically diagnosed with ATR-X syndrome. Our study shows the preponderance of differentially methylated genes within, or adjacent to, pericentromeric or telomeric chromosomal regions, suggesting a major role of heterochromatin in the pathophysiology of ATR-X, linked to the disruption of ATRX function in the context of its role as a regulator of heterochromatin dynamics.

\section{Results}

The epi-signature identified in blood samples from ATR-X patients

The genome-wide DNA methylation array of 20 blood samples obtained from ATR-X patients was compared with a reference cohort (controls). Various methylation changes at a single-probe level were identified across the genome, consisting of both hypo- and hypermethylation (estimate value $> \pm 0.15$ ) (Fig. 1a). Hierarchical clustering of significant probes $(p<0.01)$ clearly demonstrated a unique methylation profile and subclustering for these patients compared with our large laboratory reference cohort (Fig. 1c). The global methylation analysis revealed an increase in methylation at low methylation value regions (0.1-0.2 methylation value) in patients relative to controls (Fig. 1b). This pattern suggests that increased methylation is taking place in normally unmethylated regions, majority of which are normally located in promoters and $\mathrm{CpG}$ islands.

Statistical filtering to identify regions with most robust methylation changes, using multiple parameters including $p$ value $<0.01, F$ value $>50$, number of consecutive probes $>4$ and methylation difference $> \pm 20 \%$, revealed 16 genetic regions with significant statistical difference between ATR-X and controls (Table 1). Of these, 13 regions showed hypermethylation (methylation difference higher than +0.2$)$ and three regions showed hypomethylation (methylation difference lower than -0.2). These regions were distributed across the genome both outside $(n=5)$ and within CpG islands $(n=11)$, including seven regions at gene promoter $\mathrm{CpG}$ islands and two intragenic $\mathrm{CpG}$ islands (Table 1). This epi-signature was specific to ATR-X patients and did not correlate with the type of mutation at the ATRX gene locus.

We then performed a single-patient analysis to identify possible patient-specific, as opposed to patient cohortspecific, recurring methylation changes, using statistical parameters of $p<0.01$, methylation difference $> \pm 15 \%$, 

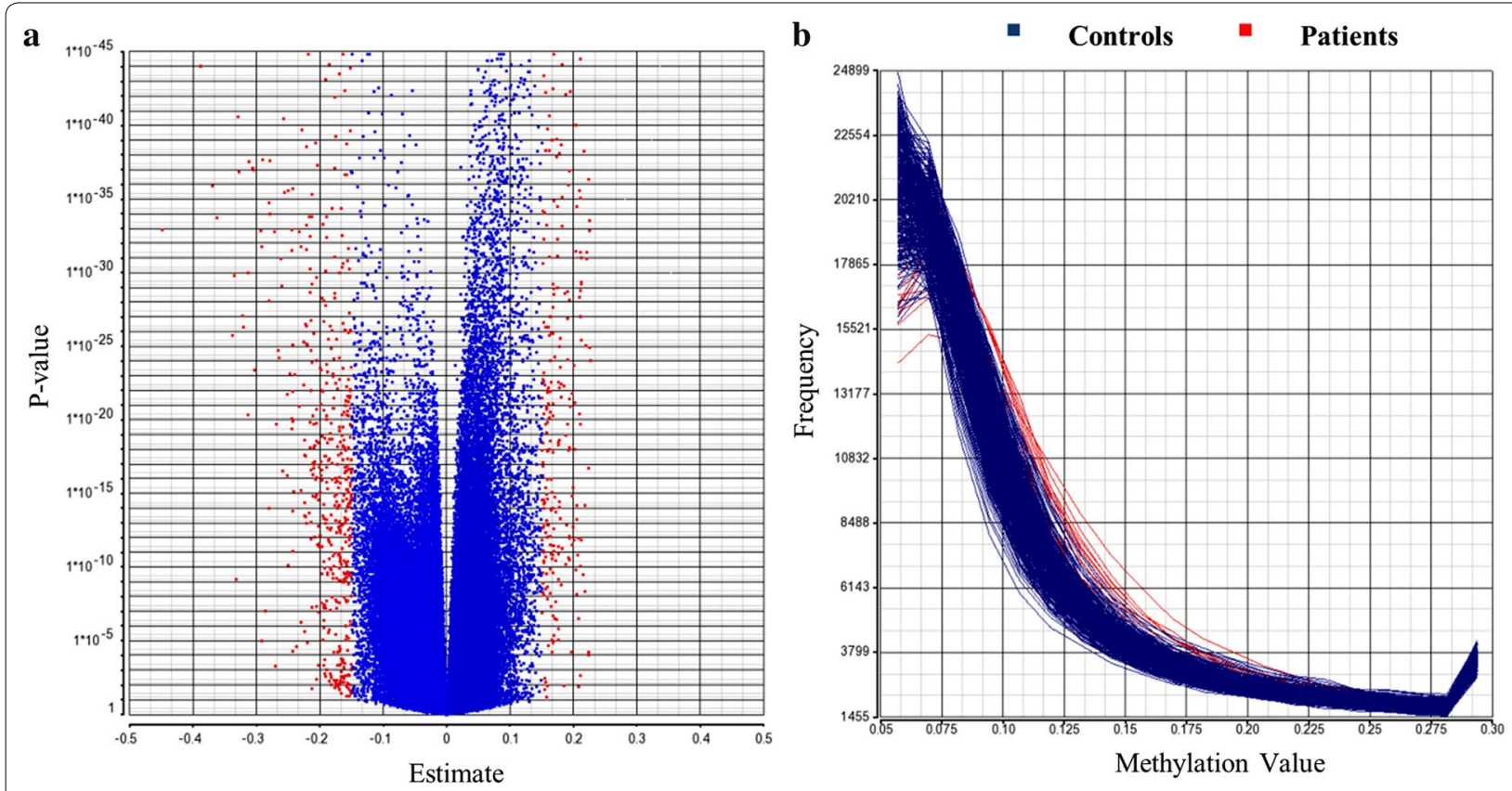

c

Controls

ATR-X
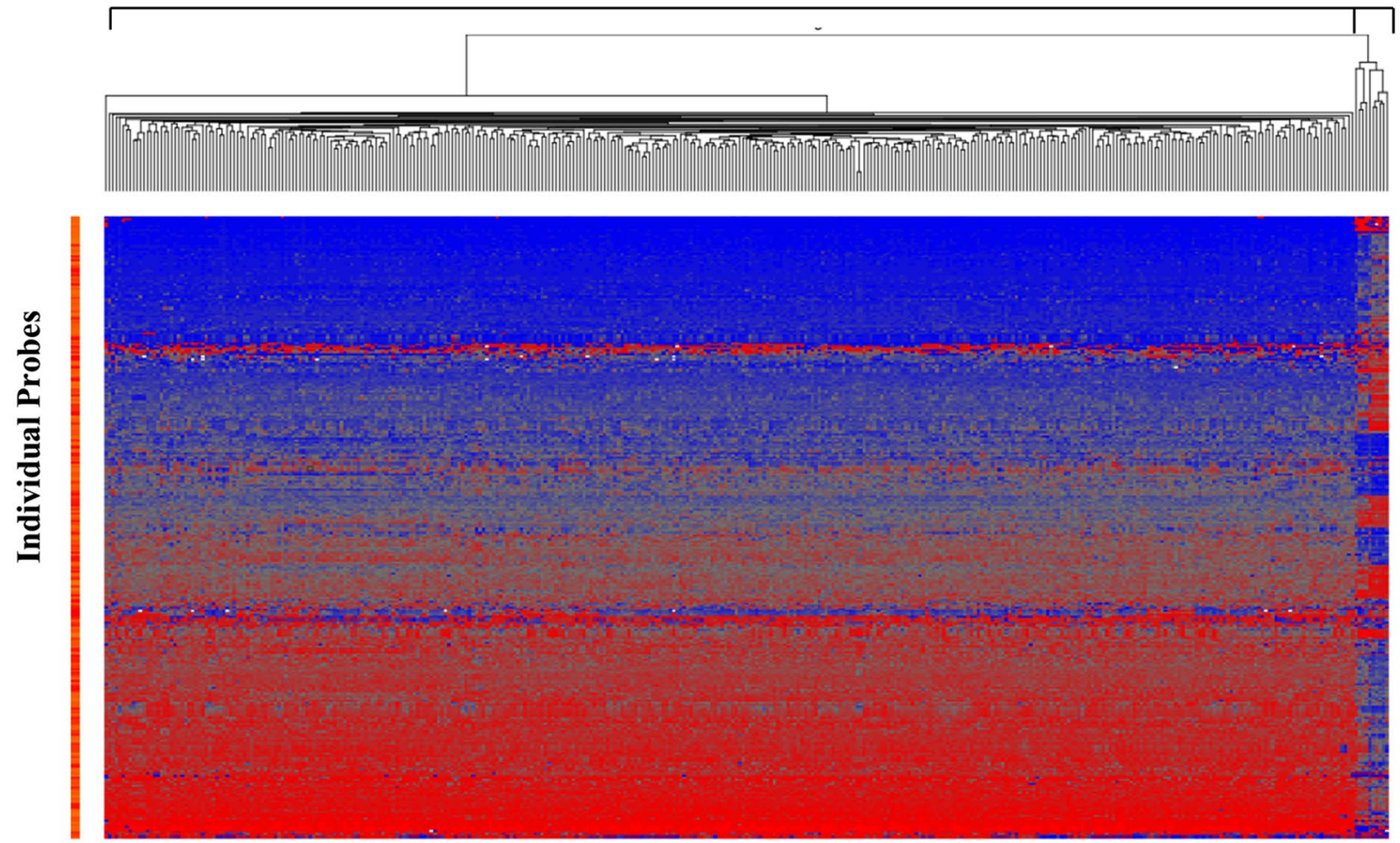

Column \# @ Minimum $\square$ Maximum

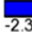

0.00

Fig. 1 a Volcano plot of methylation difference (estimate) between ATR-X and controls versus statistical significance (log $p$ value) of individual probes. In red are highlighted probes with estimate value higher than 0.15 . Positive estimate value $=$ hypermethylation; negative estimate value = hypomethylation. $\mathbf{b}$ Histogram of all samples showing methylation value ( $X$-axis) versus frequency of methylation levels across the genome (Y-axis) in ATR-X patients (red) and controls (blue). Patients with ATR-X showed a higher frequency of methylation at low methylation value regions (0.1-0.2 methylation value). Low methylation value regions are characteristic of promoter $\mathrm{CpG}$ islands. c Hierarchical clustering of probes differentially methylated $(p<0.01)$ between ATR-X and controls demonstrating marked asymmetry of the two groups. Cases are represented in the columns and probes in the rows 
Table 1 ATR-X methylation signature: significant regions detected by methylation array in ATR-X patients $(n=17)$ compared with controls $(n=210)$

\begin{tabular}{|c|c|c|c|c|c|c|c|}
\hline Chr & Region start & Region stop & \# Probes & Nearest gene & $\begin{array}{l}\text { Distance to gene } \\
\text { (bp) }\end{array}$ & $\begin{array}{l}\text { Within CpG } \\
\text { island }\end{array}$ & Gene promoter/intragenic \\
\hline chr1 & 228291476 & 228291715 & 5 & C1orf35 & 453 & No & - \\
\hline chr3 & 107810351 & 107810801 & 6 & CD47 & 415 & Yes & - \\
\hline chr3 & 109056339 & 109056907 & 4 & DPPA4 & 0 & No & Promoter \\
\hline chr4 & 124222 & 125122 & 5 & ZNF718 & 0 & Yes & Intragenic/promoter \\
\hline chr 5 & 23506728 & 23507762 & 13 & PRDM9 & 0 & No & promoter \\
\hline chr5 & 134073420 & 134073589 & 5 & CAMLG & 581 & No & - \\
\hline chr5 & 150284292 & 150284806 & 9 & ZNF300 & 0 & Yes & Promoter \\
\hline chr5 & 150325572 & 150326872 & 13 & ZNF300P1 & 0 & Yes & Promoter \\
\hline chr6 & 168045258 & 168045898 & 5 & LOC401286 & 21624 & No & - \\
\hline chr6 & 34498908 & 34499514 & 5 & PACSIN1 & 0 & Yes & Intragenic \\
\hline chr8 & 43131250 & 43132517 & 9 & POTEA & 15068 & Yes & - \\
\hline chr10 & 38069509 & 38069955 & 4 & ZNF248 & 0 & Yes & Intragenic \\
\hline chr16 & 10479552 & 10480299 & 6 & ATF7IP2 & 0 & Yes & Promoter \\
\hline chr19 & 12305543 & 12306507 & 7 & LOC100289333 & 0 & Yes & Promoter \\
\hline chr20 & 623079 & 623431 & 4 & SRXN1 & 3837 & Yes & - \\
\hline chrY & 21664286 & 21665031 & 4 & BCORP1 & 0 & Yes & Promoter \\
\hline
\end{tabular}

Significant regions: Human reference genome $\mathrm{Hg} 19$. Probes $>4$, estimate (net methylation difference) $> \pm 20 \%, F$ value $>50, p$ value $<0.01$. Gene promoters were defined as any sequence immediately surrounding the annotated $5^{\prime}$ end of the gene

across four consecutive probes. First we observed that the cohort-specific epi-signature was absent in one of the patients (patient \#12). A follow-up assessment showed that although this patient has previously been identified to carry a possible mutation in the $A T R X$ gene, more recent data demonstrated that c.5579A>G; p.N1860S in the ATRX gene is indeed a benign polymorphism and hence this patent did not have the ATR-X syndrome. The remaining 17 patients with molecular diagnosis of ATR$\mathrm{X}$, using the above statistical cutoffs, showed an average of 9.8 significant loci from the epi-signature per individual, with the minimum of four significant loci observed in two patients (\#13 and \#15). To evaluate the specificity of this assay, we applied the ATR-X epi-signature in randomly selected 15 individuals, which included normal controls that were not part of the discovery cohort, as well as individuals with Fragile X syndrome, PraderWilli syndrome, Angelman syndrome and BeckwithWiedemann syndrome. The majority of these individuals did not present any statistical significant changes at the ATRX epi-signature loci. Six control individuals showed significant changes at 1 or 2 ATRX epi-signature loci (POTEA and PACSIN1). These two genes had slightly higher level of variable DNA methylation and were as a result removed from the final ATRX epi-signature.

\section{Biological interpretation}

A more comprehensive gene list with methylation changes in the ATR-X group passing the following criteria, minimum of three consecutive probes with $p<0.01, F$ value across the region $>50$ and methylation difference $> \pm 15 \%$ (i.e., at least a $15 \%$ methylation difference), showed an overrepresentation of genes involved in biosynthetic processes, nucleic acid metabolic processes and methylation process (Table 2). Many of the genes are involved in transcriptional regulation: PRDM9 encodes a histone $\mathrm{H} 3$ lysine-4 trimethyltransferase [23]; CTDP1 functions in recruiting RNA polymerase to DNA promoters and is an OMIM gene for congenital cataracts, facial dysmorphism and neuropathy [24]; TFB2M regulates mtDNA transcription and maintenance [25]; also ZNF300, ZNF274 and ATF7IP2 are transcriptional regulators [26-28]. Another gene, QKI, regulates RNA splicing, export of target RNAs from the nucleus, translation of proteins and RNA stability [29]. In addition, three genes encode proteins associated with methylation process, including the histone $\mathrm{H} 3$ lysine-4 trimethyltransferase, a known target of ATRX and the betaine-homocysteine methyltransferase 2-BHMT2, which catalyzes the methylation of homocysteine. Evidences suggest that ATRX functions as a high-affinity RNA-binding protein and may regulate RNA stability of function [30]. These findings further support previous evidences, suggesting that ATRX has a role in regulating DNA and RNA metabolism and stability, as well as in the epigenetic regulation. Mutation at the $A T R X$ gene may result in transcriptional deregulation of several genes across the genome and consequently 
Table 2 Biological pathways identified by pathway analysis of the differentially methylated genes in ATR-X

\begin{tabular}{|c|c|c|c|c|c|}
\hline \multirow{2}{*}{$\begin{array}{l}\text { Biological pathway } \\
\text { Number of genes in group }\end{array}$} & \multicolumn{2}{|c|}{ Biosynthetic process } & \multicolumn{2}{|c|}{ Nucleic acid metabolic process } & \multirow{2}{*}{$\begin{array}{l}\text { Methylation process } \\
3\end{array}$} \\
\hline & 14 & & 12 & & \\
\hline Fisher's exact enrichment score $-\operatorname{In}(p$ value) & 5.9 & & 4.6 & & 4.6 \\
\hline Fisher's exact right-tail $p$ value & $<0.01$ & & 0.01 & & 0.01 \\
\hline \multirow[t]{7}{*}{ Genes } & DPPA4 & PPAP2C & ZNF274 & ATF7IP2 & PRDM9 \\
\hline & ZNF718 & ZNF486 & CTDP1 & ZNF486 & BHMT2 \\
\hline & PRDM9 & ZNF274 & TFB2M & ZNF300 & TFB2M \\
\hline & BHMT2 & TFB2M & QKI & DPPA4 & \\
\hline & ZNF300 & ATF7IP2 & ZNF718 & ZNF248 & \\
\hline & QKI & CTDP1 & UBE2U & PRDM9 & \\
\hline & ZNF248 & ALG10B & & & \\
\hline
\end{tabular}

Pathway analysis was performed with differentially methylated regions using cutoff of estimate $> \pm 0.15, p<0.01, F>50$ and probes $>3$

neurodevelopmental problems associated with the disorder.

\section{Technical validation of the methylation array}

To technically confirm the methylation array findings, we performed bisulfite mutagenesis/sequencing for two regions identified by the array: CD47 and ZNF300P1 (Fig. 2; Additional file 1). The methylation array at these regions showed hypermethylation in ATR-X patients compared with controls (Fig. 2), with an average methylation value for the $6 \mathrm{CD} 47$ probes of 0.55 and 0.8 in controls and patients (1.45 fold increase), respectively, and average methylation for the 12 probes at ZNF300P1 of 0.3 and 0.54 in controls and patients (1.8 fold increase), respectively. Using bisulfite mutagenesis/sequencing, we detected an overall increase in methylation at these regions in patients as compared to controls (Fig. 2). The bisulfite analysis showed average methylation at CD47 gene of 0.19 and 0.31 in controls and patients (1.63 fold increase), respectively, and at ZNF300P1 gene of 0.16 and 0.25 in controls and patients ( 1.56 fold increase), respectively. These findings confirm the accuracy and specificity of the DNA methylation array data.

\section{Uneven distribution of altered methylation sites across the genome}

ATRX has multiple functions related to maintenance of heterochromatin and genomic integrity that are essential during mammalian development. Hence, we hypothesized that genes in the ATRX epi-signature may be clustered in highly heterochromatinized pericentromeric and telomeric regions of the chromosomes. Using a statistical cutoff of $p<0.01$, estimate $> \pm 0.15, F$ value $>50$ and probes $>3$, the top 40 genes were localized using Karyogram view (Partek GS). We found that $17 / 40(42.5 \%)$ of these genes in our signature mapped to telomeric and subtelomeric regions (defined as the last full cyto-band region on the chromosome) and $8 / 40(20 \%)$ to pericentromeric regions (defined as the full cyto-band region on the centromere of the chromosome), with the remaining 15 (37.5\%) scattered throughout the genome (Fig. 3). These findings suggest that ATRX dysfunction has a functional consequence to genes localized within heterochromatinized regions of the genome, with potentially inappropriate gene expression of these genes/epi-signature secondary to loss of ATRX as a regulator of chromatin integrity.

\section{Discussion}

The interplay between ATRX and DNA methylation has been evidenced by early studies in EBV-transformed cells from patients with ATR-X and controls, showing that mutations at the ATRX gene cause changes in the pattern of methylation at subtelomeric and rDNA sequences [20]. Furthermore, loss of ATRX expression has been linked to extensive epigenomic alteration including CPG island hypermethylation observed in astrocytic tumors $[31,32]$. The involvement of ATRX in the regulation of DNA methylation was further supported by the discovery that ATRX interacts with MeCP2 and cohesion subunits in the brain [33, 34]. MeCP2 is a methyl-CpG-binding domain protein with affinity for GC-rich sequences and methylated DNA which in turn facilitates the recruitment of histone modifiers and chromatin remodeling complexes [35]. Similar to ATRX, MeCP2 is essential for neurodevelopment and mutations or duplications of the $\mathrm{MeCP} 2$ gene cause Rett syndrome, a neurodevelopmental disorder [36]. In addition, cohesin proteins play a role in the regulation of chromosome organization and gene expression by binding to unmethylated CTCF-associated regions and mutations at cohesin genes are associated with the developmental defects seen in patients with Cornelia de Lange syndrome [37]. MeCP2 was shown to recruit the helicase domain of ATRX to heterochromatic 


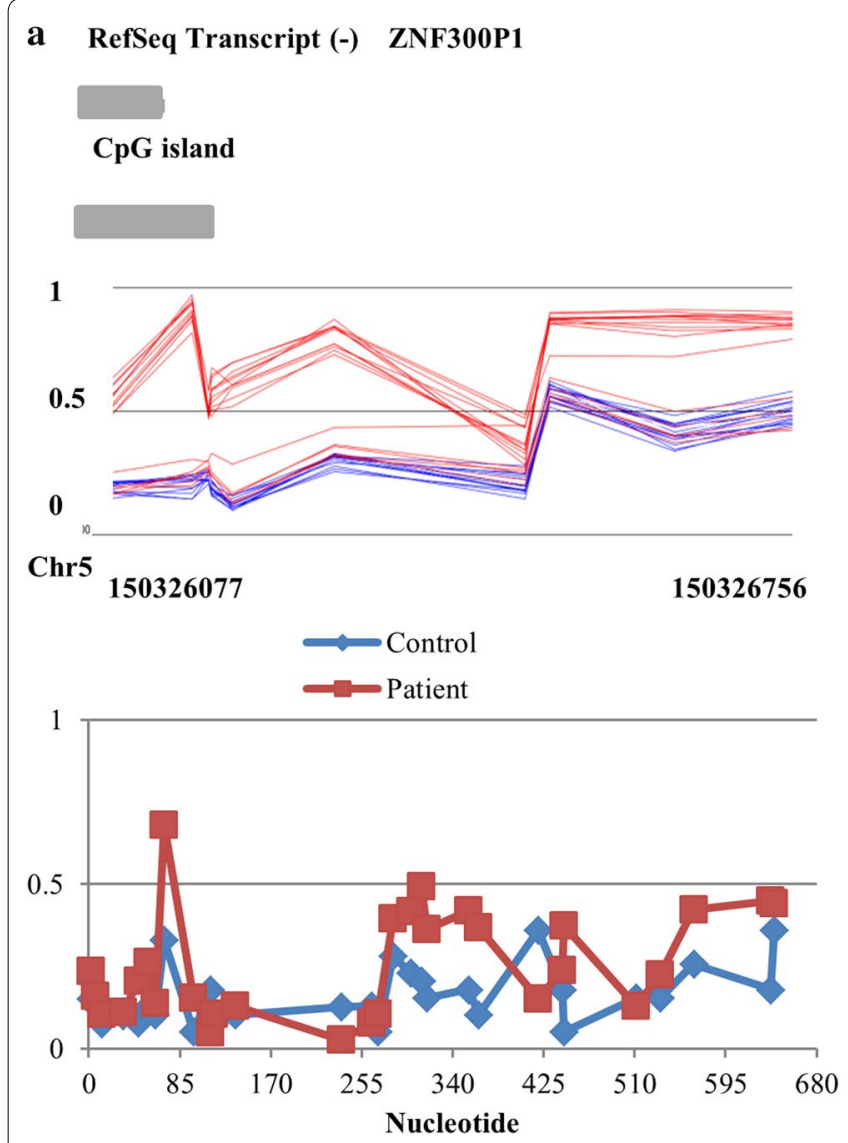

b

\section{RefSeq Transcript (-) CD47}

\section{CpG island}

1

0.5

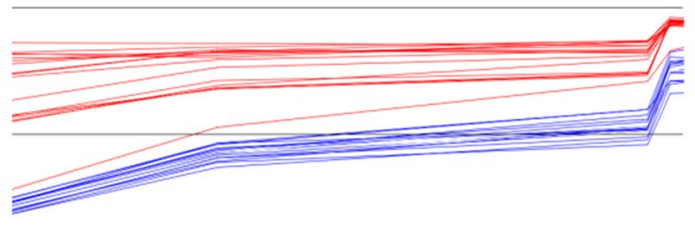

0

Chr3 107810422

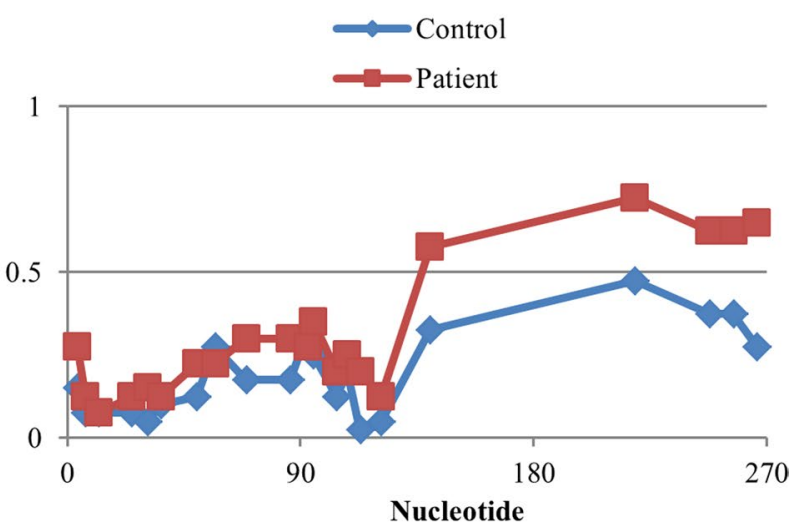

Fig. 2 Methylation visualization of significantly altered genes ZNF300P1 (a) and CD47 (b) in ATR-X patients (red) and controls (blue) identified by methylation array (top images) and bisulfite mutagenesis sequencing (bottom images). Top and bottom images show the same genomic coordinates. Methylation array figures were generated using Genomic Browser viewer (Partek) and shows methylation level 0 (not methylated) to 1 (100\% methylated). CpG island and gene location and chromosome coordinates are also represented. The bottom image corresponds to methylation average based on bisulfite sequencing data from two ATR-X patients and two controls across the same regions from the top image. Bisulfite mutagenesis and sequencing analysis of these regions confirms effects seen by methylation array analysis

regions in a DNA methylation-dependent manner [33]. In addition, MeCP2 has been reported to interact with DNA methyltransferase 1 in order to perform maintenance methylation in vivo [38], as well as with histone $\mathrm{H} 3$ lysine 9 methyltransferase enzymes, to reinforce a repressive chromatin state by bridging DNA methylation and histone methylation [39].

Both ATRX protein and de novo DNA methyltransferases DNMT3A/B/L contain a histone-binding domain (ADD) that has been shown to play a role in the establishment and maintenance of DNA methylation patterns. The ADD domain interacts with specific methylation modifications of histone lysine 4 and 9 (H3K4 and H3K9). The $\mathrm{H} 3 \mathrm{~K} 4$ methylation is associated with gene transcription and promoters DNA hypomethylation, whereas methylation of $\mathrm{H} 3 \mathrm{~K} 9$ is a heterochromatin-associated mark associated with transcriptional repression and DNA hypermethylation [16, 17]. H3K4 and H3K9 methylation is proposed to act as chromatin-based signals for regulation of DNA methylation, while ATRX-heterochromatin interaction depends on these histone methylation markers [16]. ATRX ADD domain binds to the methylated H3K9 (H3K9me3) in conjunction with unmodified H3K4 which are commonly seen in the repressed repeat elements. Therefore, mutations that functionally disrupt the ATRX protein and result in "mis-targeting" of ATRXheterochromatin interaction may provide a mechanism for abnormal DNA methylation patterns in patients with the ATR-X syndrome. While ATRX does not contain a DNA methyltransferase domain, we and others [20] have clearly shown association between ATRX mutation and abnormal patterns of DNA methylation.

The mechanism for ATRX induction of DNA methylation aberrations is not well known. Evidence has shown 


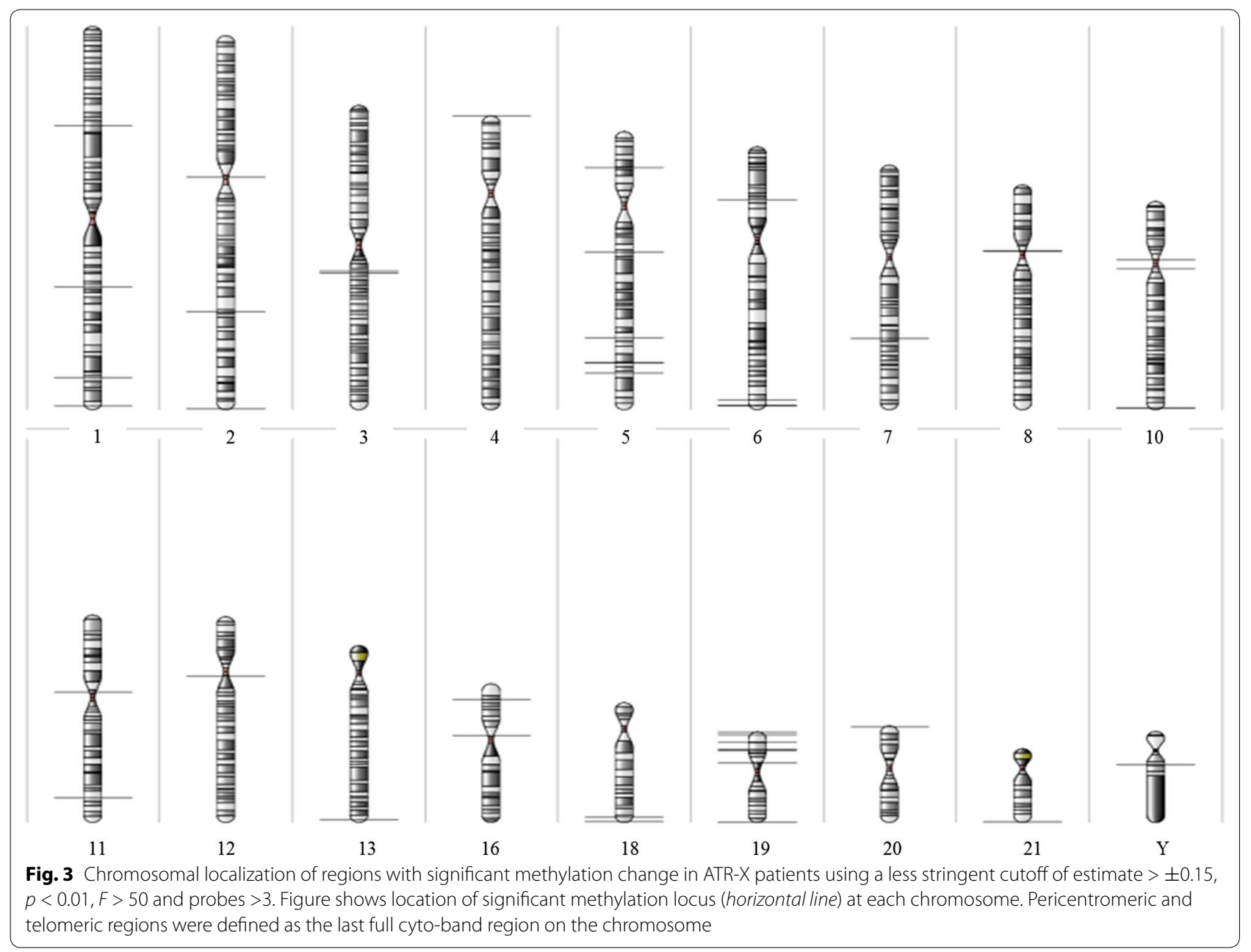

an overlapping function of ATRX and ATF7IP2. A genome-wide promoter DNA methylation study, using methylation-dependent immunoprecipitation-Chip assay, has demonstrated hypermethylation at ATF7IP2 gene in patients with ATRX mutation [40]. ATF7IP2, also known as MBD1-containing chromatin-associated factor 2, is known to bind to the transcription repression domain of the methylated cytosine-binding protein MBD1, as well as to interact with the H3K9 methyltransferase SETDB1 [28]. The overlapping protein interaction of ATRX-ATF7IP2 suggests that they form part of the same repressive chromatin complex, which involves ATF7IP2-induced H3K9me3 and ATRX binding to H3K9me3. There is also evidence for ATF7IP2 and ATRX transcriptional activation role, through SP1 and DAXX interaction, in promyelocytic leukemia nuclear bodies $[28,41,42]$. These data suggest that ATRX and ATF7IP2 have overlapping repressive/activating chromatin remodeling properties and potentially function in overlapping gene regulation pathways.
Most studies assessing the regulation of DNA methylation by ATRX have been focused on repetitive sequences and gene-specific methylation analysis. A recent study using methylation-sensitive restriction endonuclease has shown that ATRX mutations are associated with alterations in the DNA methylation profiles in highly repetitive sequences [20]. Another study using bisulfite mutagenesis analysis in mice model has demonstrated that specific gene activation at ancestral pseudoautosomal regions, which are repetitive sequences regulated by ATRX, does not involve gene-specific changes on DNA methylation, but relies on the ATRX-dependent H3.3 deposition mechanism [43]. However, none of these studies have analyzed global DNA methylation and/or specific gene CpG islands methylation in non-repetitive sequences. By using a high-resolution methylation array technique and a large reference cohort (controls), our study has clearly shown the existence of a pattern of DNA methylation changes, including in promoter $\mathrm{CpG}$ islands, telomeric and pericentromeric regions, in patients with ATR-X. 
Accordingly, in our study, most of the DNA methylation changes observed in patients with an ATRX gene mutation were localized at telomeric and pericentromeric regions. How the epigenetic consequences of ATRX mutations actually result in the disease phenotype is not well understood. It is possible that the methylation alterations could result in differences in transcriptional regulation. For example, hypomethylation in a gene promoter $\mathrm{CpG}$ island may result in increased transcription, whereas hypermethylation may result in decreased transcriptional activity [6]. Gene pathway analysis showed that many of the genes identified in the ATR-X epi-signature are associated with DNA and RNA metabolic process, which may be involved in the regulation of specific gene expression and corroborate to the cardinal developmental processes disrupted in this rare disease; however, further research involving integrative analysis of gene expression and DNA methylation profiling to investigate the relationship between these DNA methylation changes and gene expression is warranted.

Here, we propose that the most significant and recurrent regions altered in the genomic DNA of patients with ATR-X, consisting of 14 loci, provide an epigenetic signature for this syndrome which may be used as a high sensitive and specific diagnostic biomarker to support the diagnosis of ATR-X, particularly in patients with phenotypical complexity and/or with $A T R X$ gene sequence variants of unknown significance. Previous findings have demonstrated evidence of loss of DNA methylation in the repetitive elements [20]. While theoretically, it would be possible to use repetitive element methylation patterns as part of a unique ATRX mutation epi-signature, routine analysis of the repetitive elements DNA methylation pattern can be challenging due to the lack of specificity for assays designed for assessment of methylation of genomic repeats. Furthermore, most array or sequencing-based bisulfate protocols are limited to targeting unique genomic sequences. Therefore, identification of a robust unique epigenetic signature across a large number of unique genetic sequences that we describe in this manuscript presents an opportunity for utilization of these findings in routine clinical diagnostics.

In addition to the ATR-X epi-signature described here, our group has recently demonstrated unique DNA methylation signatures in patients with two other conditions, including Floating-Harbor syndrome, which is caused by mutation in the SRCAP gene, as well as cerebellar ataxia, deafness and narcolepsy syndrome, which is caused by mutations in the DNMT1 gene $[44,45]$. Other groups have also identified epi-signatures in patients with Sotos syndrome [46], and the X-linked intellectual disability caused by the $K D M 5 C$ gene [47]. Taken together, these studies demonstrate the ability of genome-wide methylation array to accurately diagnose multiple epigenetic disorders. Utilization of this technology in routine clinical practice will enable the discovery of new epigenetic biomarkers and will serve to enhance our understanding of human disease etiology. However, the identification of epigenetic variants of unknown clinical significance (E-VUS) will require delivery of testing to be performed in regulated clinical laboratories along with an adequate control cohort of normal samples, together with the development and implementation of clinical and laboratory testing guidelines, and availability and integration with pre- and posttest genetic counseling.

\section{Conclusion}

In conclusion, the observation of genome-wide epigenetic defects in ATR-X patients expands our understanding of the pathology of this disease, in which specific DNA methylation changes could lead directly to an aberrant expression of a number of genes in ATRX-deficient patients, particularly, but not restricted to telomeric and pericentromeric regions, thus contributing to the phenotypes associated with ATR-X syndrome. In addition, the unique epi-signature identified for ATR-X syndrome can now be used as an epigenetic biomarker to support the diagnosis of new patients using a sensitive, specific and cost-effective GWMA testing protocol.

\section{Methods}

\section{Sample collection, DNA extraction and genotyping}

Peripheral blood samples from patients referred for genetic testing at the Greenwood Genetic Center were collected for methylation study. All patients were consented and counseled for ATRX testing as part of their clinical referral. Ethical approval was consented by the Self Regional Healthcare Institutional Review Board (IRB \#26). Genomic DNA was extracted from peripheral blood leukocyte using standard techniques. Patients presenting with the alpha thalassemia/mental retardation X-linked syndrome (ATR-X) underwent molecular diagnostic confirmation (ATRX gene analysis) and were selected for the methylation study. Table 3 shows the molecular (mutations) characteristics of these patients. The ATR-X panel of patients is composed of 18 males with average age of 12.2 years (ranging from $8 \mathrm{~m}$ to 27 years).

The methylation array of these patients was compared with a reference cohort composed of controls and individuals previously referred for microarray with no significant methylation alteration. The reference cohort (controls) is composed of 210 male controls with average age of 7.3 years ( $2 \mathrm{~m}-53$ years). 
Table 3 Clinical and molecular characteristics of ATR-X patients referred for methylation study

\begin{tabular}{|c|c|}
\hline ATR-X patient no. & Mutation \\
\hline 1 & c.109C>T; p.R37X \\
\hline 2 & c.109C>T; p.R37X \\
\hline 3 & c.109C>T; p.R37X \\
\hline 4 & c.109C>T; p.R37X \\
\hline 5 & c.109C>T; p.R37X \\
\hline 6 & c.730A $>C ; p .1244 \mathrm{~L}$ \\
\hline 7 & c.758T>C; p.L253S \\
\hline 8 & c.736T>C; p.R246C \\
\hline 9 & C.736C>T; P.R246C \\
\hline 10 & c.952G>T; p.G249C \\
\hline 11 & c.4817G>A; p.S1606N \\
\hline 12 & c.5579A>G; p.N1860S \\
\hline 13 & c.5786A>G; p.K1929R \\
\hline 14 & c.6254G>A; p.R2085H \\
\hline 15 & c.6593A>G; p.H2198R \\
\hline 16 & c.7156C>T; p.R2386X \\
\hline 17 & c.7366_7367 InsA; p.M2456Nfs X42 \\
\hline 18 & Deletion of exon 2 \\
\hline
\end{tabular}

\section{Methylation array and data analysis}

The DNA methylation array was performed using the Infinium HumanMethylation450 BeadChip (Illumina) according to standard protocol at the Genetic and Molecular Epidemiology Laboratory at McMaster University. The array coverage includes $>485,000$ individual methylation sites, $99 \%$ of RefSeq genes and $96 \%$ of CpG islands. Beta and intensity values for methylation were generated using the Illumina Genome Studio Software, and .idat files were imported to Partek Genomic Suite software (Partek GS). The patient cohort was compared with the laboratory reference cohort. Statistical analysis was performed to compare ATR-X patients versus the control cohort using the ANOVA test to generate probe-level statistics, including $p$ value $(t$ test), $F$ value (signal to noise) and estimate value (net methylation difference). The cutoff of estimate $> \pm 0.20, p<0.01$, $F>50$ and probes $>4$ was used to select the top significant regions to be included in the epi-signature. A less stringent cutoff of estimate $> \pm 0.15, p<0.01, F>50$ and probes $>3$ was used for pathway analysis and karyogram view in order to include a larger number of regions in those analysis. Significant regions were mapped against the CpG islands and gene promoter regions. Genomic visualization of the data was performed using the karyogram view toll (Partek GS) for chromosome distribution of differentially methylated regions, and the Genomic Browser Wizard (Partek GS) for locus-specific methylation levels.

\section{Pathway analysis}

The top 45 differentially methylated genes identified using a less stringent cutoffs (Additional file 2) were assessed using the pathway analysis tool in the Partek Genomics Suite software. Briefly, statistical analysis included Fisher's exact test and was restricted to functional groups at least two genes. Results show the enrichment $p$ value ( $p$ value of the Fisher exact test reflective of the number of the genes in versus not in the list or functional group) and the enrichment score (negative log of the enrichment $p$ value; a high score indicates that the genes in the functional group are overrepresented in the gene list).

\section{Bisulfite mutagenesis}

Genomic DNA isolated from blood of ATR-X patients $(n=2)$ and controls $(n=2)$ was sodium bisulfite treated using the EZ DNA Methylation-Direct Kit (Zymo Research) according to manufacturer's instructions. DNA was amplified by nested PCR and the resulting products ligated into the pGEM-T Easy vector using a TOPO-TA cloning kit (Invitrogen). Positive clones were sequenced with Applied Biosystem 3730xl DNA Analyzer technology (Center for Applied Genomics, McGill University). Clones were accepted at $\geq 95 \%$ conversion. Non-converted cytosine residues and mismatched base pairs were used to ensure all clones originated from unique template DNA.

\section{Additional files}

Additional file 1: Figure S1. Methylation string diagrams of significantly altered regions in ATR-X patients and controls. Bisulfite mutagenesis and sequencing analysis was performed in approximately 20 alleles from each sample, and individual alleles are represented as a string of CpGs. The total average methylation for each sample is indicated. Unmethylated CpGs are represented as empty circles, and methylated CpGs as filled circles.

Additional file 2: Table S1. ATR-X methylation; significant regions detected by methylation array in ATR-X patients $(n=17)$ compared with controls $(n=210)$ using cutoff of probes $>3$, estimate $>15 \%$, F value $>50$, $p$ value $<0.01$

\section{Abbreviations}

ATRX: alpha thalassemia/mental retardation X-linked protein; ATR-X: alpha thalassemia/mental retardation X-linked syndrome; DAXX: death domain-associated protein; DNMT: DNA methyltransferase; ES cells: embryonic stem cells; E-VUS: epigenetic variants of unknown clinical significance; GWMA: genomewide DNA methylation array; H3K4: histone 3 lysine 4; H3K9: histone 3 lysine 9; KDM5C: lysine (K)-specific demethylase 5C; MeCP2: methyl CpG-binding protein 2; Partek GS: Partek Genomic Suite software; PCR: polymerase chain reaction; SRCAP: Snf2-related CREBBP activator protein; SWI/SNF: SWItch/ sucrose non-fermentable family; TR: tandem repeats.

\section{Authors' contributions}

LCS analyzed the methylation data and was a major contributor in writing the manuscript. KDK and AM performed bisulfite methylation analysis. DR and $\mathrm{AH}$ performed the methylation microarray. PJA, NGB and DIR contributed to data interpretation and manuscript writing. KMB, GP and BS supervised the 
methylation study and contributed to manuscript writing. C Schwartz and C Skinner provided the samples and clinical data for the study. All authors read and approved the final manuscript.

\begin{abstract}
Author details
${ }^{1}$ Department of Pathology and Lab Medicine, Western University, London, ON, Canada. ${ }^{2}$ Children's Hospital of Eastern Ontario Research Institute, University of Ottawa, Ottawa, ON, Canada. ${ }^{3}$ Molecular Genetics Laboratory, Victoria Hospital, London Health Sciences Center, 800 Commissioner's Road E, B10-104, London, ON N6A 5W9, Canada. ${ }^{4}$ Department of Paediatrics, Western University, London, ON, Canada. ${ }^{5}$ Department of Biochemistry, Western University, London, ON, Canada. ${ }^{6}$ Department of Oncology, Western University, London, ON, Canada. ${ }^{7}$ Children's Health Research Institute, London, ON, Canada. ${ }^{8}$ Department of Pathology and Molecular Medicine, McMaster University, Hamilton, ON, Canada. ${ }^{9}$ Department of Clinical Epidemiology and Biostatistics, McMaster University, Hamilton, ON, Canada. ${ }^{10}$ Center for Molecular Studies, J.C. Self Research Institute of Human Genetics, Greenwood Genetic Center, Greenwood, SC, USA.
\end{abstract}

\section{Acknowledgements}

We thank the patients and their families for their participation in studies conducted by the Greenwood Genetic Center. Dedicated to the memory of Ethan Francis Schwartz (1996-1998).

\section{Competing interests}

The authors declare that they have no competing interests.

\section{Availability of data and materials}

Methylation data can be provided upon request.

\section{Ethical approval and consent to participate}

The Self Regional Healthcare Institutional Review Board approved the project (IRB \#26). All patients provided a written informed consent before their inclusion in the study in accordance with the Declaration of Helsinki.

\section{Funding}

This project was supported in part by a Grant from the South Carolina Department of Disabilities and Special Needs (SC DDSN). This work was also supported by the Care4Rare Canada Consortium (Enhanced Care for Rare Genetic Diseases in Canada) funded by Genome Canada, the Canadian Institutes of Health Research, the Ontario Genomics Institute, Ontario Research Fund, Genome Quebec and Children's Hospital of Eastern Ontario Foundation.

Received: 7 November 2016 Accepted: 1 March 2017

Published online: 10 March 2017

\section{References}

1. Bjornsson HT. The Mendelian disorders of the epigenetic machinery. Genome Res. 2015;25:1473-81. doi:10.1101/gr.190629.115.

2. Rodenhiser $D$, Mann M. Epigenetics and human disease: translating basic biology into clinical applications. CMAJ. 2006;174:341-8. doi:10.1503/ cmaj.050774

3. Jones PA. Functions of DNA methylation: islands, start sites, gene bodies and beyond. Nat Rev Genet. 2012;13:484-92. doi:10.1038/nrg3230.

4. Tan M, Luo H, Lee S, Jin F, Yang JS, Montellier E, Buchou T, Cheng Z, Rousseaux S, Rajagopal N, et al. Identification of 67 histone marks and histone lysine crotonylation as a new type of histone modification. Cell. 2011;146:1016-28. doi:10.1016/j.cell.2011.08.008.

5. Shen H, Laird PW. Interplay between the cancer genome and epigenome. Cell. 2013;153:38-55. doi:10.1016/j.cell.2013.03.008.

6. Schenkel LC, Rodenhiser DI, Ainsworth PJ, Pare G, Sadikovic B. DNA methylation analysis in constitutional disorders: clinical implications of the epigenome. Crit Rev Clin Lab Sci. 2016;53:147-65. doi:10.3109/10408 363.2015.1113496.

7. Gibbons R. Alpha thalassaemia-mental retardation, X linked. Orphanet J Rare Dis. 2006;1:15. doi:10.1186/1750-1172-1-15.

8. Voon HP, Gibbons RJ. Maintaining memory of silencing at imprinted differentially methylated regions. CMLS. 2016;73:1871-9. doi:10.1007/ s00018-016-2157-6.
9. Watson LA, Goldberg H, Berube NG. Emerging roles of ATRX in cancer. Epigenomics. 2015;7:1365-78. doi:10.2217/epi.15.82.

10. Pickett HA, Reddel RR. Molecular mechanisms of activity and derepression of alternative lengthening of telomeres. Nat Struct Mol Biol. 2015;22:875-80. doi:10.1038/nsmb.3106.

11. Watson LA, Solomon LA, Li JR, Jiang Y, Edwards M, Shin-ya K, Beier F, Berube NG. Atrx deficiency induces telomere dysfunction, endocrine defects, and reduced life span. J Clin Investig. 2013;123:2049-63. doi:10.1172/JCl65634.

12. Ritchie K, Seah C, Moulin J, Isaac C, Dick F, Berube NG. Loss of ATRX leads to chromosome cohesion and congression defects. J Cell Biol. 2008;180:315-24. doi:10.1083/jcb.200706083.

13. Kernohan KD, Vernimmen D, Gloor GB, Berube NG. Analysis of neonatal brain lacking ATRX or MeCP2 reveals changes in nucleosome density, CTCF binding and chromatin looping. Nucleic Acids Res. 2014;42:835668. doi:10.1093/nar/gku564.

14. De La Fuente R, Viveiros MM, Wigglesworth K, Eppig JJ. ATRX, a member of the SNF2 family of helicase/ATPases, is required for chromosome alignment and meiotic spindle organization in metaphase II stage mouse oocytes. Dev Biol. 2004;272:1-14. doi:10.1016/j.ydbio.2003.12.012.

15. Leung JW, Ghosal G, Wang W, Shen X, Wang J, Li L, Chen J. Alpha thalassemia/mental retardation syndrome $X$-linked gene product ATRX is required for proper replication restart and cellular resistance to replication stress. J Biol Chem. 2013;288:6342-50. doi:10.1074/jbc.M112.411603.

16. Noh KM, Allis CD, Li H. Reading between the lines: "ADD"-ing histone and DNA methylation marks toward a new epigenetic "Sum". ACS Chem Biol. 2016;11:554-63. doi:10.1021/acschembio.5b00830.

17. Goldberg AD, Banaszynski LA, Noh KM, Lewis PW, Elsaesser SJ, Stadler S, Dewell S, Law M, Guo X, Li X, et al. Distinct factors control histone variant H3.3 localization at specific genomic regions. Cell. 2010;140:678-91. doi:10.1016/j.cell.2010.01.003.

18. Voon HP, Hughes JR, Rode C, De La Rosa-Velazquez IA, Jenuwein T, Feil $R$, Higgs DR, Gibbons RJ. ATRX plays a key role in maintaining silencing at interstitial heterochromatic loci and imprinted genes. Cell Rep. 2015;11:405-18. doi:10.1016/j.celrep.2015.03.036.

19. Udugama M, Chang FTM, Chan FL, Tang MC, Pickett HA, McGhie JD, Mayne L, Collas P, Mann JR, Wong LH. Histone variant H3.3 provides the heterochromatic $\mathrm{H} 3$ lysine 9 tri-methylation mark at telomeres. Nucleic Acids Res. 2015;43:10227-37. doi:10.1093/nar/gkv847.

20. Gibbons RJ, McDowell TL, Raman S, O'Rourke DM, Garrick D, Ayyub $H$, Higgs DR. Mutations in ATRX, encoding a SWI/SNF-like protein, cause diverse changes in the pattern of DNA methylation. Nat Genet. 2000;24:368-71. doi:10.1038/74191.

21. Law MJ, Lower KM, Voon HP, Hughes JR, Garrick D, Viprakasit V, Mitson M, De Gobbi M, Marra M, Morris A, et al. ATR-X syndrome protein targets tandem repeats and influences allele-specific expression in a size-dependent manner. Cell. 2010;143:367-78. doi:10.1016/j. cell.2010.09.023.

22. Ratnakumar K, Bernstein E. ATRX: the case of a peculiar chromatin remodeler. Epigenetics. 2013;8:3-9. doi:10.4161/epi.23271.

23. Mihola O, Trachtulec Z, Vlcek C, Schimenti JC, Forejt J. A mouse speciation gene encodes a meiotic histone $\mathrm{H} 3$ methyltransferase. Science. 2009;323:373-5. doi:10.1126/science.1163601.

24. Archambault J, Pan G, Dahmus GK, Cartier M, Marshall N, Zhang S, Dahmus ME, Greenblatt J. FCP1, the RAP74-interacting subunit of a human protein phosphatase that dephosphorylates the carboxyl-terminal domain of RNA polymerase IIO. J Biol Chem. 1998;273:27593-601.

25. Cotney J, McKay SE, Shadel GS. Elucidation of separate, but collaborative functions of the rRNA methyltransferase-related human mitochondrial transcription factors B1 and B2 in mitochondrial biogenesis reveals new insight into maternally inherited deafness. Hum Mol Genet. 2009;18:2670-82. doi:10.1093/hmg/ddp208.

26. Gou D, Wang J, Gao L, Sun Y, Peng X, Huang J, Li W. Identification and functional analysis of a novel human $\mathrm{KRAB} / \mathrm{C} 2 \mathrm{H} 2$ zinc finger gene ZNF300. Biochim Biophys Acta. 2004;1676:203-9.

27. Yano K, Ueki N, Oda T, Seki N, Masuho Y, Muramatsu M. Identification and characterization of human ZNF274 CDNA, which encodes a novel Kruppel-type zinc-finger protein having nucleolar targeting ability. Genomics. 2000;65:75-80. doi:10.1006/geno.2000.6140.

28. Ichimura T, Watanabe S, Sakamoto Y, Aoto T, Fujita N, Nakao M. Transcriptional repression and heterochromatin formation by MBD1 and MCAF/ 
AM family proteins. J Biol Chem. 2005;280:13928-35. doi:10.1074/jbc. M413654200.

29. Lauriat TL, Shiue L, Haroutunian V, Verbitsky M, Ares M Jr, Ospina L, Mclnnes $L A$. Developmental expression profile of quaking, a candidate gene for schizophrenia, and its target genes in human prefrontal cortex and hippocampus shows regional specificity. J Neurosci Res. 2008;86:785-96. doi:10.1002/jnr.21534.

30. Sarma K, Cifuentes-Rojas C, Ergun A, Del Rosario A, Jeon Y, White F, Sadreyev R, Lee JT. ATRX directs binding of PRC2 to Xist RNA and Polycomb targets. Cell. 2014;159:869-83. doi:10.1016/j.cell.2014.10.019.

31. Turcan $S$, Rohle D, Goenka A, Walsh LA, Fang F, Yilmaz E, Campos C, Fabius AW, Lu C, Ward PS, et al. IDH1 mutation is sufficient to establish the glioma hypermethylator phenotype. Nature. 2012;483:479-83. doi:10.1038/ nature10866.

32. Cai J, Yang P, Zhang C, Zhang W, Liu Y, Bao Z, Liu X, Du W, Wang H, Jiang T, Jiang C. ATRX mRNA expression combined with IDH1/2 mutational status and Ki-67 expression refines the molecular classification of astrocytic tumors: evidence from the whole transcriptome sequencing of 169 samples samples. Oncotarget. 2014;5:2551-61. doi:10.18632/oncotarget.1838.

33. Nan X, Hou J, Maclean A, Nasir J, Lafuente MJ, Shu X, Kriaucionis S, Bird A. Interaction between chromatin proteins MECP2 and ATRX is disrupted by mutations that cause inherited mental retardation. Proc Natl Acad Sci USA. 2007;104:2709-14. doi:10.1073/pnas.0608056104.

34. Kernohan KD, Jiang Y, Tremblay DC, Bonvissuto AC, Eubanks JH, Mann MR, Berube NG. ATRX partners with cohesin and MeCP2 and contributes to developmental silencing of imprinted genes in the brain. Dev Cell. 2010;18:191-202. doi:10.1016/j.devcel.2009.12.017.

35. Portela A, Esteller M. Epigenetic modifications and human disease. Nat Biotechnol. 2010;28:1057-68. doi:10.1038/nbt.1685.

36. LaSalle JM, Yasui DH. Evolving role of MeCP2 in Rett syndrome and autism. Epigenomics. 2009;1:119-30. doi:10.2217/epi.09.13.

37. Watrin E, Kaiser FJ, Wendt KS. Gene regulation and chromatin organization: relevance of cohesin mutations to human disease. Curr Opin Genet Dev. 2016;37:59-66. doi:10.1016/j.gde.2015.12.004.

38. Kimura H, Shiota K. Methyl-CpG-binding protein, $\mathrm{MeCP} 2$, is a target molecule for maintenance DNA methyltransferase, Dnmt1. J Biol Chem. 2003;278:4806-12. doi:10.1074/jbc.M209923200.
39. Fuks F, Hurd PJ, Wolf D, Nan X, Bird AP, Kouzarides T. The methyl-CpGbinding protein MeCP2 links DNA methylation to histone methylation. J Biol Chem. 2003;278:4035-40. doi:10.1074/jbc.M210256200.

40. Carvill GaS A. Genome-wide DNA methylation analysis in patients with familial ATR-X mental retardation syndrome. In: Appasani K, editor. Epigenomics from chromatin biology to therapeutics, vol. 1. New York: Cambridge University Press; 2012. p. 434-46.

41. Xue Y, Gibbons R, Yan Z, Yang D, McDowell TL, Sechi S, Qin J, Zhou S, Higgs D, Wang W. The ATRX syndrome protein forms a chromatinremodeling complex with Daxx and localizes in promyelocytic leukemia nuclear bodies. Proc Natl Acad Sci USA. 2003;100:10635-40. doi:10.1073/ pnas. 1937626100.

42. Vallian S, Chin KV, Chang KS. The promyelocytic leukemia protein interacts with Sp1 and inhibits its transactivation of the epidermal growth factor receptor promoter. Mol Cell Biol. 1998;18:7147-56.

43. Levy MA, Kernohan KD, Jiang Y, Berube NG. ATRX promotes gene expression by facilitating transcriptional elongation through guanine-rich coding regions. Hum Mol Genet. 2015;24:1824-35. doi:10.1093/hmg/ddu596.

44. Hood RL, Schenkel LC, Nikkel SM, Ainsworth PJ, Pare G, Boycott KM, Bulman DE, Sadikovic B. The defining DNA methylation signature of Floating-Harbor Syndrome. Sci Rep. 2016;6:38803. doi:10.1038/srep38803.

45. Kernohan KD, Cigana Schenkel L, Huang L, Smith A, Pare G, Ainsworth $P$, Boycott KM, Warman-Chardon J, Sadikovic B. Identification of a methylation profile for DNMT1-associated autosomal dominant cerebellar ataxia, deafness, and narcolepsy. Clin Epigenetics. 2016;8:91. doi:10.1186/ s13148-016-0254-X.

46. Choufani S, Cytrynbaum C, Chung BH, Turinsky AL, Grafodatskaya D, Chen YA, Cohen AS, Dupuis L, Butcher DT, Siu MT, et al. NSD1 mutations generate a genome-wide DNA methylation signature. Nat Commun. 2015;6:10207. doi:10.1038/ncomms10207.

47. Grafodatskaya D, Chung BH, Butcher DT, Turinsky AL, Goodman SJ, Choufani S, Chen YA, Lou Y, Zhao C, Rajendram R, et al. Multilocus loss of DNA methylation in individuals with mutations in the histone H3 lysine 4 demethylase KDM5C. BMC Med Genomics. 2013;6:1. doi:10.1186/1755-8794-6-1.

\section{Submit your next manuscript to BioMed Central and we will help you at every step:}

- We accept pre-submission inquiries

- Our selector tool helps you to find the most relevant journal

- We provide round the clock customer support

- Convenient online submission

- Thorough peer review

- Inclusion in PubMed and all major indexing services

- Maximum visibility for your research

Submit your manuscript at www.biomedcentral.com/submit
() Biomed Central 